\title{
Childhood Immunizations in Nepal: Present Situation and Future Directions.
}

\author{
Adhikari N. ${ }^{1}$ \\ 1. Dr. Neelam Adhikari. Chief of Pediatric Services, Patan Hospital. Professor of Pediatrics, National Academy of Medical Sciences \\ (NAMS).
}

Address for correspondence: neelamadhikari@gmail.com

Immunization prevents millions of deaths every year and reduces the costs of treatment and disability caused by infectious diseases. Immunization also has the potential to significantly boost economic growth. ${ }^{1}$ By improving the health of a population, immunization also improves their education and work prospects. Despite political disturbances and insurgency in the last twelve years, it is heartening to note a very good childhood immunization coverage all over the country in Nepal. Nepal Demographic and Health Survey 2006, reports excellent coverage by age 12 months $^{2}$. Eighty percent of children had all basic vaccines. Eighty percent of children had received measles vaccine; and that only $3.2 \%$ of children had not been immunized. It is also a noteworthy fact that because of additional measles immunization provided to children less than 14 years of age; the incidence of measles has come down markedly. In addition, achievements in the field of polio eradication are also praiseworthy. If it was not for the two bordering states of India(Bihar and UP), Nepal may have been certified a polio free country.

The field of immunization is now extremely exciting. This is especially true in the countries where basic immunization delivery systems are well in place. Yet in many countries, efforts to increase immunization coverage are hampered by high cost of some of the vaccines. As a result more than 28 million children missed out on immunization during their first year of life- leaving them vulnerable to infectious diseases both during childhood and during the productive adult years; and more than 1.5 million children die every year in the world's poorest regions from rotavirus and pneumococcal diseases for which newly licensed vaccines are available ${ }^{3}$.

For poor countries like Nepal, Global Alliance for Vaccine and Immunization (GAVI) a public private global health partnership, launched in 2000 has already moved into its second phase (2006-15). Nepal is already a beneficiary; Hepatitis B vaccine is now provided through routine immunizations all over the country. World Health Organization's (WHO) position paper on Haemophilus influenza type $b$ conjugate vaccines has affirmed safety and efficacy of conjugate Hib vaccines. It has recommended that this vaccine should be included in all routine infant immunization programmes. It is further recommended that lack of local surveillance data should not delay the introduction of this vaccine $e^{4}$. It is once again heartening to note that Hib vaccine will be introduced in immunization programme of Nepal by 2009.
A number of other new vaccines are or will soon be available which could prevent many more childhood deaths. We will be soon talking about new Pneumococcal, Rotavirus, and Human Papilloma vaccines for Nepal. Once introduced in industrialized countries, it takes an average of 15-20 years for a new vaccine to become widely available in the developing world. Advance Market Commitments (AMC) will help to minimize these gaps ${ }^{5}$. AMC for vaccines is a financial commitment to subscribe the future purchase (for a certain pre determined price) for a vaccine not yet available if an appropriate vaccine is developed and if it demanded by developing countries. An AMC is not a purchase guarantee, as industry will receive the subsidy if the product meets the targeted standards and there is demand for these products. An AMC has been designed for pneumococcal vaccines.

With such exciting developments, we from NEPAS need to update our policies, guidelines and recommendations for childhood immunizations. Some suggestions for this purpose are as below.

Universal immunizations for children must include the boosters of diphtheria and tetanus. MMR vaccines should be incorporated in national immunization schedule. First dose of measles vaccination at 9 months and a dose of MMR vaccine at 15 months of age will obviate need for National Measles Immunization campaigns. However, while considering strategy of rubella immunization of children, simultaneous immunization of child bearing age women should be adopted to decrease incidence of congenital rubella syndrome.

\section{References:}

1. David Bloom, David Canning and Mark Weston: The value of immunization, World Economics Vol 6, no 3, July-Sept 2005

2. Ministry of Health, New Era, Macro International: Nepal Demographic and Health Survey 2006

3. WHO data 2005

4. WHO Position Paper on Hemophilus Influenzae vaccines: Weekly Epidemiological Record, 24 November 2006, $81^{\text {st }}$ year.

5. Advance Market Commitments as posted on website: www.gavialliance.org January 2007 